\title{
An improved $k-\varepsilon$ turbulence model for FENE-P fluids capable to reach high drag reduction regime
}

\author{
P.R. Resende ${ }^{a, *}$, A.M. Afonso ${ }^{\mathrm{b}}$, D.O. Cruz ${ }^{\mathrm{c}}$ \\ a São Paulo State University (UNESP), Institute of Science and Technology, Sorocaba, Brazil

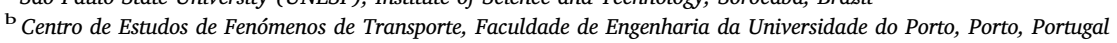 \\ ${ }^{\mathrm{c}}$ Programa de Engenharia Mecânica (DEM/COPPE/UFRJ), Rio de Janeiro, Brazil
}

\section{A R T I C L E I N F O}

\section{Keywords:}

Isotropic turbulence model

Drag reduction

FENE-P fluids

\begin{abstract}
A B S T R A C T
An improved $k-\varepsilon$ turbulence model for viscoelastic fluids is developed to predict turbulent flows in complex geometries, with polymeric solutions described by the finitely extensible nonlinear elastic-Peterlin constitutive model. The $k-\varepsilon$ model is tested against a wide range of direct numerical simulation data, with different rheological parameters combinations, and is capable to capture the drag reduction for all regimes of low, intermediate and high, with good performance. Two main contributions are proposed, one through the viscoelastic closures present in the turbulent kinetic energy and dissipation equations, and the other, by modifying eddy viscosity model damping function to incorporate the viscoelastic effect close to the wall, especially at the buffer layer. In addition, improvements have been made to the cross-correlations between the fluctuating components of the polymer conformation and rate of strain tensors present in the Reynolds-averaged transport equation for the conformation tensor. The main advantage is the capacity to predict all components of the tensor with good performance.
\end{abstract}

\section{Introduction}

The drag reduction (DR) phenomenon present in turbulent viscoelastic flows has been studied for decades. It is general knowledge that the addition of polymeric particles in a Newtonian fluid in a turbulent flow it reach up to $80 \%$ reduction in transport energy, by reducing the turbulent friction. Another effect is the reduction in the heat transfer, both phenomena provide a significant advantage in terms of energy reduction for thermal energy transportation at long distances, and for this reason researchers dedicate their efforts to use it in engineering systems, such as district heating systems.

After the discovery of the drag reduction phenomenon, several studies were carried out to understand the origin of the drag reduction in turbulent flows, and several theories have been proposed to describe this complex mechanism, but no definitive consensus has been reached. Two preferred theories are the Lumley theory (Lumley, 1969 and Lumley, 1973), the so-called viscous idea, and the Tabor and De Gennes theory (Tabor and Gennes, 1986), known as the elastic explanation. Lumley assumes that the DR phenomenon is a consequence of an increase of the effective viscosity in a region outside of the viscous sublayer and in the buffer layer, caused by the polymer stretching within a turbulent flow. It also assumes that the beginning of drag reduction occur when the time scale of the polymers overcome the time scale of the flow. The other assumption of Tabor and De Gennes is that the elastic energy stored by the polymer becomes equivalent to the turbulent kinetic energy in the buffer layer, and inhibits the usual energy transfer and thickens the buffer layer, where the viscoelastic length scale is larger than the Kolmogorov scale.

It is generally accepted that DR is linked to the increase of the effective elongational viscosity of a dilute polymer solution, caused by the full coil-stretch transition of the elastic polymer molecules in the direction of the mean flow, leading to a reduction in the vortex dynamic activities, according to the Lumley theory. Latter, L'vov et al. (2004) quantified the viscous scenario by showing the additional effective viscosity increasing linearly with the distance from the wall in the buffer layer, and also proposed a direct numerical simulations (DNS) of Newtonian turbulent flows including an artificial viscosity to confirm its theory. This was performed by De Angelis et al. (2004), which obtained the same drag reducing properties of the equivalent viscoelastic FENE-P simulations (Angelis et al., 2002).

However, DNS results between the two scenarios showed some contradictions, when considering the high and maximum drag reduction regimes. Recently, Dallas et al. (2010) demonstrated that high drag reduction could be achieved without full coil-stretch transition of the

\footnotetext{
* Corresponding author.

E-mail address: resende@sorocaba.unesp.br (P.R. Resende).
} 
polymer molecules, in contradiction with the viscous theories. This confirms Min et al. (2003) explanation, in which the polymers in the near-wall region extract energy from the flow due to the uncoiling caused by the mean shear, and release part of the stored elastic energy back to the flow, by contracting as they shift away from the wall.

In an attempt to understand and explore the two prominent drag reducing theories, Thais et al. (2013) carried out DNS simulations at high Reynolds numbers to minimize the influence of viscous wall effects in the flow dynamics, i.e., to confirm the Lumley's viscous explanation (Lumley, 1969). The results showed to be contradictory with Dallas et al. (2010), where the primary effect of the interaction between the turbulent and polymeric fields was the opposite, transferring energy from the turbulence to the polymer. Another aspect is that the amplitude of the direct coupling between turbulent and elastic energies did not change between low and high elasticity viscoelastic flows. In resume, the interactions between the turbulent and viscoelastic energies are a complex nonlinear process, and can only be fully understood when all information on both the flow and the viscoelastic parameters are gathered (Thais et al., 2013).

Although the DNS is a powerful tool, it is not feasible for most of the engineering purposes involving turbulent viscoelastic flows, because it is significantly more expensive at a computational level, in terms of CPU-time and memory requirements, due to the larger number of primary variables. For higher DR the DNS use is even more restrictive since the near wall streaks become progressively stabilized and elongated, requiring the use of longer simulation, especially for high DR values.

An alternative approach is the used of Reynolds-averaged Navier-Stokes (RANS) models, which are less demanding computationally, and therefore gained an increased interest in the last decades. The first attempt to incorporate the elastic effect in turbulence models was made by the group of Pinho (2003) and Cruz et al. (2004), which developed a low-Reynolds numbers $k-\varepsilon$ turbulence model using Generalized Newtonian Fluid (GNF) constitutive equation, which includes the dependence of the fluid strain hardening on the third invariant of the rate of deformation tensor. They also developed an anisotropic version which includes the increased Reynolds stress anisotropy (Resende et al., 2006), and a Reynolds stress turbulence model (Resende et al., 2013), capable of predicting satisfactorily the drag reduction. However these models are limited because the modified GNF constitute equation is an inelastic model.

Due to the appearance of the DNS data of turbulent viscoelastic fluids, new developments in RANS models were possible. Leighton et al. (2003) proposed the first turbulence model for viscoelastic flows based on the FENE-P dumbbell constitutive equation model, which developed a closure to the polymer stress-strain coupling based on a tensor expansion combining the Reynolds stress and the conformation tensors. Later Pinho et al. (2008) based on a-priori analyses of DNS data, developed low-Reynolds-number $k-\varepsilon$ model for FENE-P fluids. Several closures were proposed to the terms of the governing equations, with special attention to the viscoelastic terms, the viscoelastic turbulent transport term of the turbulent kinetic energy and the non-linear term involving the conformation tensor and the strain rate fluctuations of the conformation tensor equation. This model was valid for low drag reduction regime (DR $<20 \%$ ). Subsequently, Resende et al. (2011) extended the turbulence model to intermediate drag reduction regimes, but it was not capable to reach higher values of DR. Despite the high performance of the closures, the model introduced many coefficients and damping functions, which makes it unattractive to use. Following the same procedure, Resende et al. (2013) developed a low-Reynolds-number $k$ - $\omega$ model for FENE-P fluids, showing the versatility of the previous closures in the isotropic context, but presenting the same limitations of the previous $k-\varepsilon$ model. Nevertheless the model showed to be more robust, taking the advantages of a typical $k-\omega$ turbulence model for Newtonian fluids.

Iaccarino et al. (2010) proposed a $k-\varepsilon-\overline{v^{2}}-f$ model for FENE-P fluids capable of predicting all range of DR, in a fully developed channel flow, in which they introduced the concept of turbulent polymer viscosity to account for the combined effects of turbulence and viscoelasticity on the polymer extra stress tensor term into the momentum equation. Their closure of the nonlinear term in the conformation tensor equation is simpler than the closure proposed by Resende et al. (2011) model, but is not capable to capture all components individually, only the trace of the tensor. Later, Masoudian et al. (2013) improved the model to be valid up to maximum DR, using also the concept of turbulent polymer viscosity. The main improvements consisted in the viscoelastic stress and in the viscoelastic stress work closures introduced in the momentum and turbulent kinetic energy transport equations. It also included the viscoelastic destruction term in the dissipation transport equation, following the same procedure of Resende et al. (2011).

The second order Reynolds stress model for FENE-P fluids proposed by Masoudian et al. (2015), is capable to predict all drag reduction regimes. The viscoelastic interaction in the model was reduced to the nonlinear fluctuating and destruction terms, introduced by the FENE-P and dissipation transport equations, respectively. The nonlinear viscoelastic closure was modelled involving the Reynolds stresses, similar to the Leighton et al. (2003) procedure, and the destruction viscoelastic closure was the same used by Masoudian et al. (2013). Despite the simplicity of the viscoelastic closures, the model in general is not attractive, due to the high number of Newtonian terms involved, but it can be easily overcome by the use of non-linear turbulence models.

More recently, Tsukahara and Kawaguchi (2013) proposed a low Reynolds number $k-\varepsilon$ model for viscoelastic fluids described by the Giesekus constitutive equation, using the same principle of the group of Pinho (2003) and Cruz et al. (2004). Although the model is valid up to the maximum DR, it shows some instabilities for higher Weissenberg numbers, with significant deviations from the DNS data.

In the present study, an improved $k-\varepsilon$ turbulence model for FENE-P fluids is proposed, validated for all regimes of drag reduction (low, intermediate and high) with good performance. An important contribution of this model is the development of new closures for eddy viscosity through the damping function, modified to include elastic effects, and the nonlinear fluctuating term in the conformation transport equation, which increased the stability and robustness, while keeping the same simplicity and versatility of a typically $k-\varepsilon$ turbulence model for Newtonian fluids. The model is assessed against DNS data, covering a wide range of flow conditions in terms of Weissenberg number, maximum polymer extensibility $\left(L^{2}\right)$, solvent ratio $(\beta)$ and Reynolds number, and is also compared with other FENE-P fluids closures available on the literature.

The paper is organized as follows: Section 2 introduces the instantaneous and time-averaged governing equations and identifies the viscoelastic terms that will require modelling. In Section 3, the developed turbulent closures are explained in detail, then Section 4 summaries the present model, followed by the results for the predictions at a fully developed turbulent channel flow, covering all range of DR, and finally the main conclusions are presented.

\section{Governing equations}

The exact instantaneous continuity and momentum equations for the turbulent flow of incompressible dilute polymer solution, where the rheological representation is based on the FENE-P constitutive model (Bird et al., 1980), are:

$\frac{\partial \hat{u}_{k}}{\partial x_{k}}=0$

$\rho\left(\frac{\partial \hat{u}_{i}}{\partial t}+\hat{u}_{k} \frac{\partial \hat{u}_{i}}{\partial x_{k}}\right)=-\frac{\partial \hat{p}}{\partial x_{i}}+\frac{\partial \hat{\tau}_{i k}}{\partial x_{k}}$

where the hat represents instantaneous quantities of velocity, $\hat{u}_{i}$, 
pressure, $\hat{p}$, and stress tensor, $\hat{\tau}_{i k}$, and $\rho$ the fluid density. The stress tensor, $\hat{\tau}_{i k}$, is a sum of Newtonian solvent which obeys Newton's law of viscosity, $\hat{\tau}_{i k}{ }_{i k}=2 \eta_{s} \hat{s}_{i j}$, and polymeric contributions, $\hat{\tau}^{p_{i k}}$, Eq. (3), with $\eta_{s}$ representing the solvent viscosity coefficient.

$\hat{\tau}_{i k}=\hat{\tau}^{s}{ }_{i k}+\hat{\tau}^{p_{i k}}$

The instantaneous rate of strain tensor, $\hat{s}_{i j}$, is defined as

$\hat{s}_{i j}=\frac{1}{2}\left(\frac{\partial \hat{u}_{i}}{\partial x_{j}}+\frac{\partial \hat{u}_{j}}{\partial x_{i}}\right)$

The definition of the instantaneous polymer stresses based on the FENE-P closure is given by Eq. (5) and Eq (6), function of the instantaneous conformation tensor $\hat{c}_{i j}$.

$\hat{\tau}_{i j}^{p}=\frac{\eta_{p}}{\lambda}\left(f\left(\hat{c}_{i j}\right) \hat{c}_{i j}-\delta_{i j}\right)$

with

$f\left(\hat{c}_{k k}\right)=\frac{L^{2}-3}{L^{2}-\hat{c}_{k k}}$

where $\hat{c}_{k k}$ is the trace of the instantaneous conformation tensor, and the other parameters are associated to the rheological model: $L^{2}$ denotes the maximum molecular extensibility of the dumbbell; $\lambda$ is the relaxation time of the fluid and $\eta_{p}$ is polymer viscosity coefficient. The conformation tensor behaviour obeys to the following hyperbolic differential equation

$\frac{\partial \hat{c}_{i j}}{\partial t}+\hat{u}_{k} \frac{\partial \hat{c}_{i j}}{\partial x_{k}}-\left(\hat{c}_{k j} \frac{\partial \hat{u}_{i}}{\partial x_{k}}+\hat{c}_{i k} \frac{\partial \hat{u}_{j}}{\partial x_{k}}\right)=\stackrel{\nabla}{\hat{c}_{i j}}=-\frac{\hat{\tau}_{i j}^{p}}{\eta_{p}}$

The $\hat{c}_{i j}$ term denote Oldroyd's upper convective derivative of the instantaneous conformation tensor. The first two terms are the material derivative and represent the local and advective derivatives, and the terms in the parenthesis account for the polymer stretching by the instantaneous flow.

The Reynolds average equations can be obtained by applying the Reynolds decomposition, $\hat{u}_{i}=U_{i}+u_{i}$, where the upper-case letters or overbars represent average quantities and lower-case letters or primes represent instantaneous quantities, and time-averaging, typically called Reynolds-averaged Navier-Stokes/Reynolds-averaged conformation evolution (RANS/RACE) equations:

$\frac{\partial U_{k}}{\partial x_{k}}=0$

$\rho \frac{\partial U_{i}}{\partial t}+\rho U_{k} \frac{\partial U_{i}}{\partial x_{k}}=-\frac{\partial \bar{p}}{\partial x_{i}}+\eta_{s} \frac{\partial^{2} U_{i}}{\partial x_{k} \partial x_{k}}-\frac{\partial}{\partial x_{k}}\left(\rho \overline{u_{i} u_{k}}\right)+\frac{\partial \bar{\tau}_{i k, p}}{\partial x_{k}}$

where $\bar{p}$ is the mean pressure, $U_{i}$ is the mean velocity, $-\rho \overline{u_{i} u_{k}}$ is the Reynolds stress tensor and $\bar{\tau}_{i k, p}$ the Reynolds-averaged polymer stress. Described by the FENE-P constitutive equation, the $\bar{\tau}_{i k, p}$ take the form of $\bar{\tau}_{i j, p}=\frac{\eta_{p}}{\lambda}\left[f\left(C_{k k}\right) C_{i j}-\delta_{i j}\right]+\frac{\eta_{p}}{\lambda} \overline{f\left(C_{k k}+c_{k k}\right) c_{i j}}$,

where the last term on the right side of the equation needs a closure, and the Peterlin function becomes,

$f\left(C_{k k}\right)=\frac{L^{2}-3}{L^{2}-C_{k k}}$.

Both Eq. (10) and Eq. (11), depends of the average tensor conformation, $C_{i j}$, which is determined by Reynolds average conformation evolution, Eq. (12). The different terms have specific designations, according with Li et al. (2006) and Housiadas et al. (2005).

$$
\begin{aligned}
& {[\underbrace{\frac{\partial C_{i j}}{\partial t}+U_{k} \frac{\partial C_{i j}}{\partial x_{k}}}_{D C_{i j} / D t}-\underbrace{\left(C_{j k} \frac{\partial U_{i}}{\partial x_{k}}+C_{i k} \frac{\partial U_{j}}{\partial x_{k}}\right)}_{M_{i j}}]+\underbrace{\overline{u_{k} \frac{\partial c_{i j}}{\partial x_{k}}}}_{C T_{i j}}-\underbrace{\left(\overline{c_{k j} \frac{\partial u_{i}}{\partial x_{k}}}+\overline{c_{i k} \frac{\partial u_{j}}{\partial x_{k}}}\right)}_{N L T_{i j}}} \\
& =-\frac{\bar{\tau}_{i j, p}}{\eta_{p}}
\end{aligned}
$$

The first-term inside the brackets on the left-hand-side is Oldroyd's upper convective derivative of $C_{i j}$, and the second term, $M_{i j}$, is the mean flow distortion. The remaining terms on the left-hand-side, $C T_{i j}$ and $N L T_{i j}$, account the velocity fluctuations to the advective transport of the mean conformation tensor, and the interactions between the fluctuating components of the conformation and velocity gradient tensors that originate from Oldroyd's upper convected derivative, respectively. To calculate the molecular conformation it is necessary to quantify the two terms with overbars, using adequate closures, together with the corresponding polymer stress, Eq. (10).

The closures developed in this work used DNS data to simplify and calibrate the model coefficients. The DNS data pertain to all regimes of drag reduction and are part of the large sets of data for FENE-P fluids in fully-developed turbulent channel flow, produced by Li et al. (2006), $\mathrm{Li}$ et al. (2006) and Kim et al. (2007). The non-dimensional numbers are defined as follows: the Reynolds number $R e_{\tau_{0}} \equiv h u_{\tau} / \nu_{0}$ is based on the friction velocity $\left(u_{\tau}\right)$, the channel half-height $(h)$, the zero shear-rate kinematic viscosity of the solution, which is the sum of the kinematic viscosities of the solvent and polymer $\left(\nu_{0}=\nu_{s}+v_{p}\right)$. The Weissenberg number is given by $W i_{\tau_{0}} \equiv \lambda u_{\tau}^{2} / \nu_{0}$ and the ratio between the solvent viscosity and the solution viscosity at zero shear rate is $\beta\left(\beta \equiv \nu_{s} / \nu_{0}\right)$. The three sets of DNS data used throughout this paper to characterize the elastic behaviour in each drag reduction regime, are defined by the following parameters: $R e_{\tau 0}=395, \beta=0.9$ and two different maximum extension, (1) $L^{2}=900$ for Weissenberg numbers equal to $W i_{\tau 0}=25$ and $W i_{\tau 0}=100$, corresponding to drag reductions of $18 \%$ and $37 \%$, respectively, and (2) $L^{2}=3600$ for $W e_{\tau 0}=100$, equivalent to $51 \% \mathrm{DR}$ (case A, B and C of Table 1).

The model was tested over a wide range of conditions, combining all parameters $\beta, L^{2}, R e_{\tau_{0}}$ and $W e_{\tau 0}$, and compared with the DNS (C.F. Li et al., 2006 and C.F. Li et al., 2006), and complemented by the

Table 1

\begin{tabular}{|c|c|c|c|c|c|c|c|}
\hline \multirow[t]{2}{*}{ Case } & \multirow[t]{2}{*}{ Reference } & \multicolumn{3}{|c|}{ Rheological parameters } & \multicolumn{2}{|c|}{ Drag reduction (\%) } & \multirow[t]{2}{*}{ Error (\%) } \\
\hline & & $W i_{\tau 0}$ & $\beta$ & $L^{2}$ & DNS & Present model & \\
\hline (A) & Resende et al. (2011) & 25 & 0.9 & 900 & 18 & 19.2 & 6.7 \\
\hline (B) & Resende et al. (2011) & 100 & 0.9 & 900 & 37 & 37.5 & 1.4 \\
\hline (C) & Masoudian et al. (2013) & 100 & 0.9 & 3600 & 51 & 52.2 & 2.4 \\
\hline (D) & Li et al. (2006) & 100 & 0.9 & 14,400 & 61 & 62.3 & 2.1 \\
\hline (E) & Li et al. (2006) & 200 & 0.9 & 14,400 & 75 & 67.7 & 9.7 \\
\hline
\end{tabular}

Description of the DNS parameters for turbulent channel flow at $\operatorname{Re}_{\tau 0}=395$. 
DNS data of Iaccarino et al. (2010) and Ptasinski et al. (2003), from which we used another data set to further calibrate the model, for $R e_{\tau_{0}}$ and $\beta$ variations.

As reported in Resende et al. (2011) and Masoudian et al. (2013), the nonlinear fluctuation correlation of the average polymeric stress for fully developed turbulent flow, $\overline{f\left(C_{k k}+c_{k k}\right) c_{i j}}$ in Eq. (10), can be neglected when compared with the average conformation tensor, $C_{i j}$, in both regimes of intermediate and high DR. Following the same procedure, a contribution comparison was made to the terms of the average tensor conformation equation, Eq. (12), and confirmed that the $C T_{i j}$ term can be neglected for all regimes of drag reduction, in agreement with the findings of Housiadas et al. (2005) and Li et al. (2006). The $N L T_{i j}$ term can not be neglected, since it is of significant contribution.

The Reynolds stress tensor in Eq. (9) is computed by adopting the Boussinesq turbulent stress-strain relationship,

$-\rho \overline{u_{i} u_{j}}=2 \rho v_{T} S_{i j}-\frac{2}{3} \rho k \delta_{i j}$

where $k$ is the turbulent kinetic energy, $S_{i j}$ is the mean rate of strain tensor and $\nu_{T}$ is the eddy viscosity. The $\nu_{T}$ is modelled by the typically isotropic $k-\varepsilon$ turbulence model for low Reynolds numbers, which is modified to include the elastic effect close to the wall through the damping function.

The transport equation of turbulent kinetic energy for turbulent flow of viscoelastic fluids, using the FENE-P model, Eq. (10), is given by Eq. (14) (more details can be found in Pinho et al. (2008) andResende et al. (2011)). The transport equation of $k$ contains the typically Newtonian closures found in the classical $k-\varepsilon$ turbulence models, which we adopt here to represent the solvent contribution: the molecular diffusion, the turbulent transport of $k$ by velocity and pressure fluctuations, the rate of production of $k$ and the viscous dissipation. The last two terms on right side of equation are the viscoelastic turbulent transport, $Q^{V}$, and the viscoelastic stress work, $\varepsilon^{V}$, respectively, and they represent the viscoelastic contribution which require closure models.

$$
\begin{aligned}
\rho \frac{\partial k}{\partial t}+\rho U_{i} \frac{\partial k}{\partial x_{i}}= & \frac{\partial}{\partial x_{i}}\left[\left(\eta_{s}+\frac{\rho f_{t} \nu_{T}}{\sigma_{k}}\right) \frac{\partial k}{\partial x_{i}}\right]-\rho \overline{u_{i} u_{k}} \frac{\partial U_{i}}{\partial x_{k}}-\rho\left(\tilde{\varepsilon}^{N}+D\right)+Q^{V} \\
& -\rho \varepsilon^{V}
\end{aligned}
$$

with

$D=2 v_{s}\left(\frac{d \sqrt{k}}{d x_{i}}\right)^{2}$

The Newtonian closures followed the Nagano and Hishida (1987) model, where is used a modified Newtonian rate of dissipation for $k, \tilde{\varepsilon}^{N}$, instead of the true dissipation, $\varepsilon^{N}$, in order to increase stability of the calculation. Both are related by $\varepsilon^{N}=\tilde{\varepsilon}^{N}+D$, where $D$ is given in Eq. (15). To increase the model performance, a variable turbulent Prandtl number was introduced, via function $f_{t}$, to correct turbulent diffusion near the wall, as suggested by Nagano and Shimada (1993) and Park and Sung (1995), $f_{t}=1+3.5 \exp \left(-\left(\operatorname{Re}_{T} / 150\right)^{2}\right)$ with $\operatorname{Re}_{T}=k^{2} /\left(\nu_{s} \tilde{\varepsilon}^{N}\right)$.

Similar distribution analysis had been made by Pinho et al. (2008), Resende et al. (2011) and Masoudian et al. (2013), to the viscoelastic terms of the turbulent kinetic energy equation, the viscoelastic stress work and the viscoelastic turbulent transport, at different drag reduction regimes. Initially Pinho et al. (2008) showed that the impact of $Q^{V}$ could be neglected at low regime of DR, and subsequently Resende et al. (2011) verified that the contribution of $Q^{V}$ became more significant at intermediate DR regime, and developed a closure. Although $Q^{V}$ evolution had the same amplitude of $\varepsilon^{V}$ at a high regime of $\mathrm{DR}$, the maximum value occurs at different location, in the buffer layer, where its effect is overcome by the turbulent diffusion, revealing to have small effect in the predictions and Masoudian et al. (2013) choose to neglect the $Q^{V}$ contribution, and it will be also neglected here.
The corresponding transport equation for the modified Newtonian rate of dissipation of $k$, is given by

$$
\begin{aligned}
\frac{\partial \rho \tilde{\varepsilon}^{N}}{\partial t}+\frac{\partial \rho U_{i} \tilde{\varepsilon}^{N}}{\partial x_{i}}= & \frac{\partial}{\partial x_{i}}\left[\left(\eta_{s}+\frac{\rho f_{t} \nu_{T}}{\sigma_{\varepsilon}}\right) \frac{\partial \tilde{\varepsilon}^{N}}{\partial x_{i}}\right]+f_{1} C_{\varepsilon_{1}} \frac{\tilde{\varepsilon}^{N}}{k} P_{k}-f_{2} C_{\varepsilon_{2}} \rho \frac{\tilde{\varepsilon}^{N^{2}}}{k} \\
& +\rho E+E_{\tau_{p}}
\end{aligned}
$$

with

$P_{k}=-\rho \overline{u_{i} u_{k}} \frac{\partial U_{i}}{\partial x_{k}}$ and $E=\nu_{s} \nu_{T}\left(1-f_{\mu}\right)\left(\frac{\partial^{2} U_{j}}{\partial x_{i} \partial x_{k}}\right)^{2}$

As mention before, all terms are modelled in Newtonian context, with exception of the last term, $E_{\tau_{p}}$, which represent the viscoelastic contribution to the transport equation of $\tilde{\varepsilon}^{N}$. This term acts as a destruction of the dissipation and is given by Eq. (18).

$E_{\tau_{p}}=2 \eta_{s} \frac{\eta_{p}}{\lambda\left(L^{2}-3\right)} \overline{\frac{\partial u_{i}}{\partial x_{m}} \frac{\partial}{\partial x_{k}}\left\{\frac{\partial}{\partial x_{m}}\left[f\left(C_{n n}\right) f\left(\widehat{C}_{p p}\right) c_{q q} C_{i k}\right]\right\}}$

The damping functions of Eq. (16), take into account the low Reynolds number behaviour, are $f_{1}=1$ and $f_{2}=1-0.3 \exp \left(-\left(\operatorname{Re}_{T}\right)^{2}\right)$, and the numerical values of the remaining coefficients are: $\sigma_{k}=1.1, \sigma_{\varepsilon}=1.3$, $C_{\varepsilon_{1}}=1.45$ and $C_{\varepsilon_{2}}=1.90$.

\section{Development of viscoelastic closures}

The viscoelastic closures necessary to complete the turbulence model are developed in this section, based in five sets of DNS. To obtain the conformation tensor evolution for turbulent flows, it is necessary to develop a closure to the $N L T_{i j}$ term of the Eq. (12), which account the interaction between the fluctuating components of the conformation tensor and the velocity gradient tensor. Finally, the closures developed for the viscoelastic stress work, $\varepsilon^{V}$, and the viscoelastic destruction of the Newtonian rate of dissipation, $E_{\tau_{p}}$, related to the transport equations of $k$ and its dissipation, $\tilde{\varepsilon}^{N}$, respectively, are presented. In addition, a modification is made to the damping function $f_{\mu}$ of the eddy viscosity for low Reynolds numbers, to introduce the elastic effect next to the wall, following the same procedure realized by Pinho (2003); Cruz et al. (2004); Resende et al. (2006) and Tsukahara and Kawaguchi (2013), for the development of low-Reynolds-number $k-\varepsilon$ turbulence models to predict viscoelastic flows.

The $N L T_{i j}$ closure is developed following the same assumptions of Resende et al. (2011). Based in the transport equation of $\overline{f\left(\hat{c}_{m m}\right) c_{k j} \frac{\partial u_{i}}{\partial x_{k}}}+\overline{f\left(\hat{c}_{m m}\right) c_{i k} \frac{\partial u_{j}}{\partial x_{k}}}$ derived by Pinho et al. (2008), it assumed that

$$
\begin{aligned}
& \overline{f\left(\hat{c}_{m m}\right) c_{k j} \frac{\partial u_{i}}{\partial x_{k}}}+\overline{f\left(\hat{c}_{m m}\right) c_{i k} \frac{\partial u_{j}}{\partial x_{k}}} \approx \\
& f\left(C_{m m}\right)\left(\overline{c_{k j} \frac{\partial u_{i}}{\partial x_{k}}}+\overline{c_{i k} \frac{\partial u_{j}}{\partial x_{k}}}\right)=f\left(C_{m m}\right) N L T_{i j} .
\end{aligned}
$$

Given the high complexity of the exact transport equation of the $N L T_{i j}$, more details can be found in Pinho et al. (2008) and Resende et al. (2011)

The viscoelastic closure for the $N L T_{i j}$ term is given by Eq. (20), and it was developed to allow future simulations in complex geometries, by reducing significantly the number of terms and eliminating the necessity of the wall friction velocity dependence. 

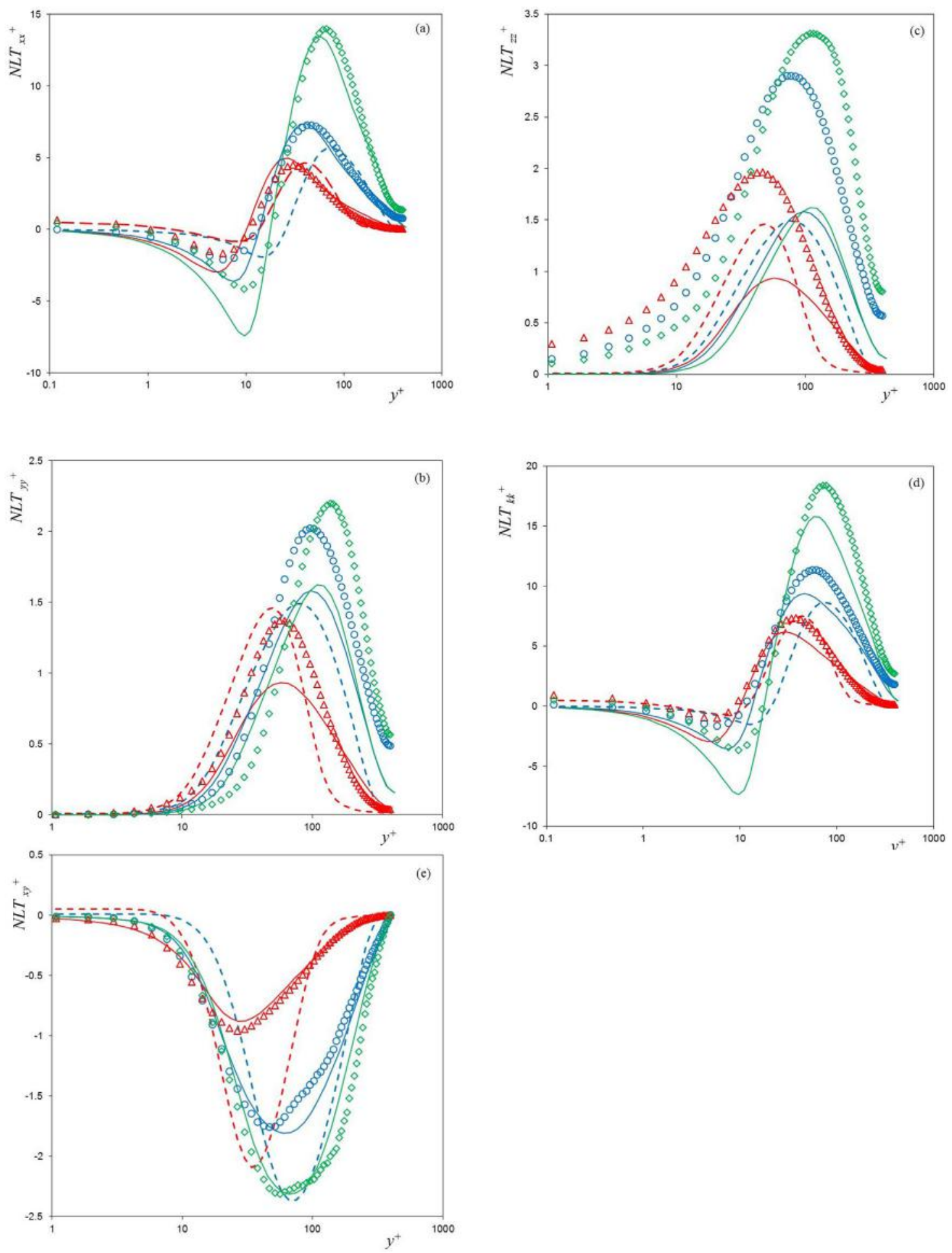

Fig. 1. Comparison of the $N L T_{i j}$ model between DNS data (symbols: $\Delta \mathrm{DR}=18 \%$, case (A); $O \mathrm{DR}=37 \%$, case (B), and $\diamond \mathrm{DR}=51 \%$, case (C)) and predictions with the new model (continuum lines), and previous model (dash lines): (a) $N L T_{x x}{ }^{+}$; (b) $N L T_{y y}{ }^{+}$; (c) $N L T_{z z}{ }^{+}$; (d) $N L T_{k k}{ }^{+}$and (e) $N L T_{x y}{ }^{+}$. Each colour corresponds to a specific DR. 


$$
\begin{aligned}
N L T_{i j} \equiv & \overline{c_{k j} \frac{\partial u_{i}}{\partial x_{k}}}+\overline{c_{i k} \frac{\partial u_{j}}{\partial x_{k}}} \approx \\
\approx & \underbrace{f_{N 1} C_{N 1} \frac{\lambda \times L^{0.42} \times \varepsilon^{N}}{v_{0} \times f\left(C_{m m}\right)^{0.8}} \delta_{i j}}_{I}-\underbrace{f_{N 1}{ }^{0.2} \frac{C_{N 2}}{L^{0.15}}\left[C_{j k} \frac{\partial U_{i}}{\partial x_{k}}+C_{i k} \frac{\partial U_{j}}{\partial x_{k}}\right]}_{I I} \\
& \underbrace{+f_{N 1}{ }^{0.9} f_{N 2} C_{N 3}\left(\frac{C_{m m} f_{\mathrm{Re}}}{(\beta / 0.9)^{0.7 \times \beta}}\right)^{0.7} \frac{\frac{\partial U_{i}}{\partial x_{k}} \frac{\partial U_{j}}{\partial x_{k}}}{\sqrt{\frac{\partial U_{p} \partial U_{p}}{\partial x_{q}}}}}_{I I I}
\end{aligned}
$$

with

$f_{N 1}=\frac{\nu_{T}}{\nu_{0}}$

$f_{N 2}=\exp \left(-\frac{f_{N 1}}{1.05 \times \sqrt{\beta} \times\left(10+0.3 L+(L / 30)-[(L / 30)-1]^{2}\right)}\right)$

$f_{\mathrm{Re}}=2-\left[1-\exp \left(-\frac{\operatorname{Re}}{3500}\right)\right]^{4}$ and $\operatorname{Re}=\frac{U_{b} \times 2 h}{v_{s}}$

where the viscoelastic coefficients are $C_{N 1}=0.02, C_{N 2}=0.6$, $C_{N 3}=0.325$.

The closure of Eq. (20) is modelled in three different parts:

- part $I$ is approached by the Taylor's longitudinal micro-scale, $\lambda_{f}$, to the relation between the double correlation of fluctuating strain rates and the turbulence kinetic energy in homogeneous isotropic turbulence,

$\frac{\overline{\partial u_{i}}}{\partial x_{k}} \frac{\partial u_{j}}{\partial x_{l}}=\frac{8}{3} \frac{k}{\lambda_{f}^{2}}\left[\delta_{i j} \delta_{k l}-\frac{1}{4}\left(\delta_{i k} \delta_{j l}+\delta_{i l} \delta_{j k}\right)\right]$

At high Reynolds number homogeneous isotropic turbulence, Taylor's longitudinal microscale is related to the dissipation of turbulent kinetic energy via equation

$\varepsilon^{N}=20 \frac{\nu_{s} k}{\lambda_{f}^{2}}$,

so according to Resende et al. (2011) the closure becomes

$$
\begin{aligned}
& \frac{\lambda}{f\left(C_{m m}\right)}\left[C_{k n}\left(\frac{\overline{\partial u_{j}}}{\partial x_{n}} \frac{\partial u_{i}}{\partial x_{k}}+\overline{\frac{\partial u_{i}}{\partial x_{n}} \frac{\partial u_{j}}{\partial x_{k}}}\right)+C_{j n} \frac{\overline{\partial u_{k}}}{\partial x_{n}} \frac{\partial u_{i}}{\partial x_{k}}+C_{i n} \frac{\overline{\partial u_{k}}}{\partial x_{n}} \frac{\partial u_{j}}{\partial x_{k}}\right] \approx, \\
& \approx \frac{\lambda}{f\left(C_{m m}\right)}\left[\frac{C_{\varepsilon_{F}}}{W e_{\tau 0}} \frac{4}{15} \times \frac{\varepsilon^{N}}{\left(\nu_{s}+\nu_{\tau P}\right) \times \beta} C_{m m} \times f_{F 2} \times \delta_{i j}\right]
\end{aligned}
$$

with $f_{F 2}=\left(1-\exp \left(-y^{+} / 25\right)\right)^{4}$ (more details can be found in Resende et al. (2011)). Here, the wall distance dependence through the damping function has been replaced by the local turbulent viscosity, $f_{N 1}=v_{T} / \nu_{0}$, and calibrated to account for $L^{2}$ variations. In addition, the dependence of the trace of the conformation tensor and the Weissenberg number was eliminated.

- part $I I$, is responsible to capture the shear component, and corresponds to the exact term, $-\left[C_{k j}\left(\partial U_{i} / \partial x_{k}\right)+C_{i k}\left(\partial U_{j} / \partial x_{k}\right)\right]$, of the approximate equation, Eq. (19), corrected by $L^{0.15}$ to considered $L^{2}$ variations, similar to the $L^{0.42}$ parameter in part $I$, and by the damping function, $f_{N 1}{ }^{0.2}$, to correct the wall effects. Also, we eliminate the $W e_{\tau 0}$ dependence, as can be observed by the term used by Resende et al. (2011), Eq. (27).

$-C_{F 2} W e_{\tau 0}^{0.743}\left[C_{k j} \frac{\partial U_{i}}{\partial x_{k}}+C_{i k} \frac{\partial U_{j}}{\partial x_{k}}\right]$

- part III is an ad-hoc term, modelled to introduce the anisotropic effect, where the $L^{2}$ and $\beta$ variations are achieved by the function $f_{N 2}$, Eq. (22), and $(\beta / 0.9)^{0.7 \times \beta}$ parameter. Also, a correction is made to low Reynolds numbers by the function $f_{\text {Re }}$, Eq. (23), consequence of the low performance of the Newtonian turbulence model at low Reynolds numbers. The approach to the wall is realized by the damping function $f_{N 1}$, Eq. (21), similar to the Masoudian et al. (2013) approximation.

An initial study was performed using only DNS data on the development of viscoelastic closures, Resende and Cavadas (2018). This model is simpler than the model of Resende et al. (2011), $N L T_{i j}$ Resende, defined by Eq. (28), and is also capable to capture all the components without the necessity of the friction velocity through the Weissenberg number.

$$
\begin{aligned}
N L T_{i j} \text { Resende } \equiv & \overline{c_{k j} \frac{\partial u_{i}}{\partial x_{k}}}+\overline{c_{i k} \frac{\partial u_{j}}{\partial x_{k}}} \\
\approx & C_{F 1}\left(0.0552 \frac{W e_{\tau 0}}{25}+0.116\right) \times \frac{C_{i j} \times f\left(C_{m m}\right)}{\lambda} \\
& -C_{F 2} W e_{\tau 0}^{0.743}\left[C_{k j} \frac{\partial U_{i}}{\partial x_{k}}+C_{i k} \frac{\partial U_{j}}{\partial x_{k}}\right] \\
+ & \frac{\lambda}{f\left(C_{m m}\right)}\left[C_{F 3} \times\left(\frac{25}{W e_{\tau 0}}\right)^{0.718}\right. \\
& \times\left(\frac{\partial U_{j}}{\partial x_{n}} \frac{\partial U_{m}}{\partial x_{k}} C_{k n} \frac{\overline{u_{i} u_{m}}}{v_{0} \sqrt{2 S_{p q} S_{p q}}}+\frac{\partial U_{i}}{\partial x_{n}} \frac{\partial U_{m}}{\partial x_{k}} C_{k n} \frac{\overline{u_{j} u_{m}}}{v_{0} \sqrt{2 S_{p q} S_{p q}}}\right. \\
+ & \left.\left.\frac{\partial U_{k}}{\partial x_{n}} \frac{\partial U_{m}}{\partial x_{k}}\left(C_{j n} \frac{\overline{u_{i} u_{m}}}{v_{0} \sqrt{2 S_{p q} S_{p q}}}+C_{i n} \frac{\overline{u_{j} u_{m}}}{v_{0} \sqrt{2 S_{p q} S_{p q}}}\right)\right)\right] \\
& -\frac{\lambda}{f\left(C_{m m}\right)} \times f_{F 1} \times C_{F 4} \times\left(\frac{25}{W e_{\tau 0}}\right)^{0.7} \\
& \times\left[C_{j n} \frac{\partial U_{k}}{\partial x_{n}} \frac{\partial U_{i}}{\partial x_{k}}+C_{i n} \frac{\partial U_{k}}{\partial x_{n}} \frac{\partial U_{j}}{\partial x_{k}}+C_{k n} \frac{\partial U_{j}}{\partial x_{n}} \frac{\partial U_{i}}{\partial x_{k}}\right. \\
& \left.+C_{k n} \frac{\partial U_{i}}{\partial x_{n}} \frac{\partial U_{j}}{\partial x_{k}}\right] \\
+ & \frac{\lambda}{f\left(C_{m m}\right)}\left[\frac{C_{\varepsilon F}}{W e_{\tau 0}} \frac{4}{15} \times \frac{\varepsilon^{N}}{\left(\nu_{s}+v_{\tau p}\right) \times \beta} C_{m m} \times f_{F 2} \times \delta_{i j}\right]
\end{aligned}
$$

The performance of the $N L T_{i j}$ closure can be analysed in Fig. 1, by comparing the predictions with the DNS data for turbulent channel flow at $R e_{\tau_{0}}=395$ and $\beta=0.9$, for different drag reduction regimes, with $L^{2}=900$ at different Weissenberg numbers, $W i_{\tau 0}=25$ and $W i_{\tau 0}=100$, equivalent to $\mathrm{DR}=18 \%$ and $37 \%$, respectively, and $L^{2}=3600$, keeping the same value of $W i_{\tau 0}=100$, corresponding to $\mathrm{DR}=51 \%$, to analyse the effect of $L^{2}$. Fig. 1(a)-(c) shows the individual normal stress components of $N L T_{i j}$ model, and the predictions are a significant improvement compared to the previous model of Resende et al. (2011), especially for higher values of DR. The $x x$ component is capable to predict well both the production and the destruction effects, close and away from the wall, respectively, capturing the maximum value and its shift away from the wall with the DR increase. In case of the $y y$ and $z z$ components, the model underpredicted the peak values due to the isotropic assumption, but all main features are well captured, such as the increase in $N L T$ with DR, the shift of the peak location to higher values of $y^{+}$as DR increases and the saturation effect. The predictions of $N L T_{i j}$ have a direct impact on the prediction of the conformation tensor, as can be observed through the Eq. (12), but in terms of the $y y$ component the Cyy is well predicted, although the underprediction of the $N L T_{y y}$ component. The correct prediction of the $C_{y y}$ is important because it contributes directly to the determination of the axial and shear component of the conformation tensor (Pinho et al., 2008), however the $z z$ component showed to had low impact. Consequently, the trace of the $N L T_{i j}$ term, Fig. 1(d), captured the main features of the normal components and underpredicted the maximum value due to the underprediction of the $y y$ and $z z$ components. The shear component of the NLT model can be observed in Fig. 1(e), where the predictions are capable to capture the behaviour with good performance for all regimes 


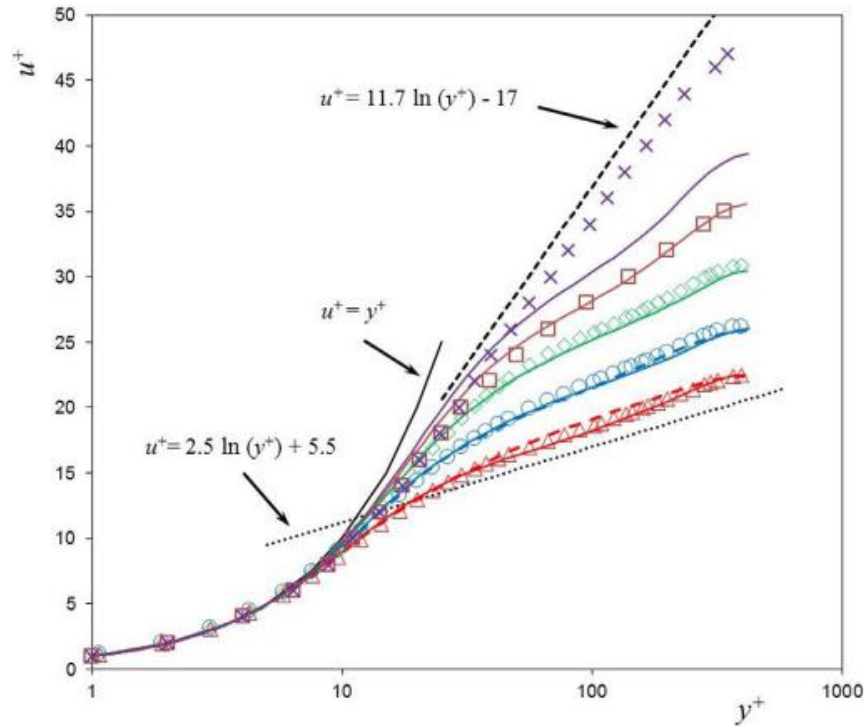

Fig. 2. Comparison of the velocity profile between DNS data (symbol: $\Delta$ $\mathrm{DR}=18 \%$; $\bigcirc \mathrm{DR}=37 \%$; $\diamond \mathrm{DR}=51 \%$; $\square \mathrm{DR}=61 \% ; \times \mathrm{DR}=75 \%$ ) and predictions, for channel turbulent flow, with rheological parameters defined in Table 1, at $\operatorname{Re}_{\tau 0}=395$ : with the new model (continuum lines), and previous model (dash lines).

of drag reduction, when compared with the DNS data.

Different attempts had been made to capture the reduction of the eddy viscosity with the DR increase, initially by Pinho et al. (2008) for low DR regime, and later by Resende et al. (2011) for intermediate DR regime, both closures presented difficulties to represent the correct behaviour, and resulted in a wrong prediction of shear Reynolds stress close to the wall, forcing the drag reduction to be achieved essential by reducing $k$. An alternative is proposed here to capture the correct behaviour of the eddy viscosity close to wall, and consequently achieved the increase of $k$ as the DR increases. The eddy viscosity is modelled considering the Newtonian solvent closure of the low Reynolds number $k-\varepsilon$ model of Nagano and Hishida (1987), given by Eq. (29), and the viscoelastic effect close to the wall is accomplished by the damping function $f_{\mu}$, Eq. (30), where the small scales redistribute the energy to the large scales of the energy cascade, Min et al. (2003). This type of approach were made by Pinho (2003); Cruz et al. (2004); Resende et al. (2006) and Tsukahara and Kawaguchi (2013) to developed viscoelastic turbulence models using different constitutive equations. The assumption made here is similar to the same principle assumed by Iaccarino et al. (2010) to develop a $k-\varepsilon-\overline{v^{2}}-f$ viscoelastic turbulence model, where the reduction of the eddy viscosity is proportional to the increase of DR, and achieved by the $\overline{v^{2}}$ reduction, since $\nu_{T}=C_{\mu} \overline{v v k} / \varepsilon$. In the present case, the increase of DR is accomplished by the trace of the conformation tensor divided by square root of the maximum molecular extensibility, $D R \sim C_{k k} / \sqrt{L^{2}}$. This assumption is a simple approximation to correct the damping function behaviour close to the wall, based on the DNS data analysis of the cases presented here. In addition, to consider the viscosity effect, the solvent ratio, $\beta$, is introduced, but as the polymeric viscosity increases the solvent ratio tends to zero, $\beta \rightarrow 0$, becoming necessary to incorporate the saturation effect by using the $\beta^{\beta}$ parameter. This parameter takes into account the variations of the polymeric component in the aqueous solution. However, its impact on the flow is not linear, because of the saturation effect. This means that the effect increases and reaches a stable value, similar to the increase of $L$, after a certain value the drag reduction does not increase and becomes stable (see Fig. 1 and Li et al., 2006). To simulate the same behaviour, the $\beta^{\beta}$ parameter was implemented in the model trough the damping function, $f_{\mu}$ and $N L T_{i j}$ model, to correct the viscoelastic behaviour near and far from the wall, respectively. Finally,

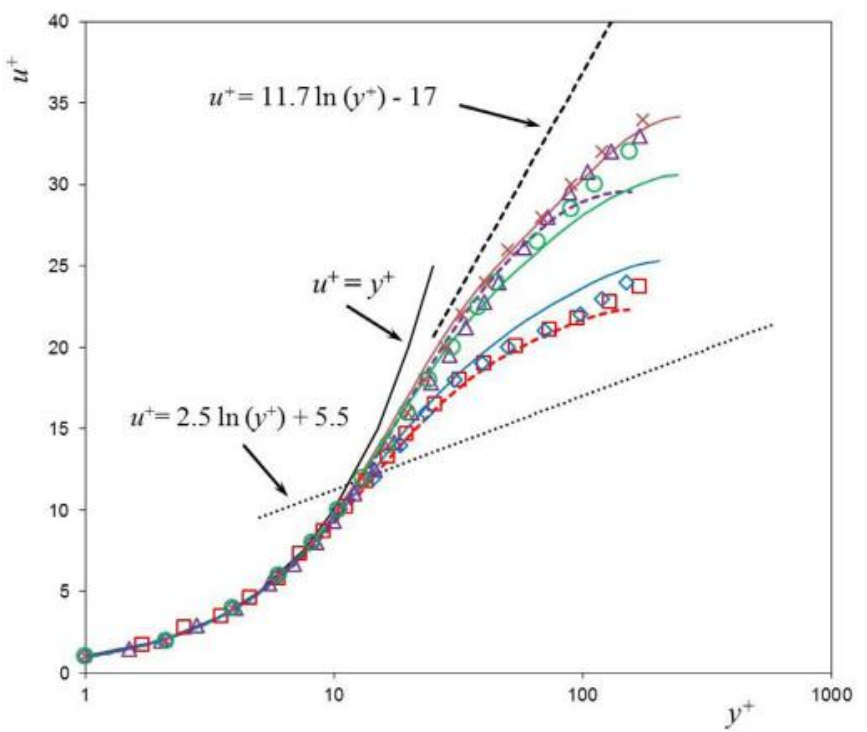

Fig. 3. Comparison of the velocity profile between DNS data from Iaccarino et al. (2010) (symbol: $\square \mathrm{DR}=33 \%$, case (F), and $\Delta \mathrm{DR}=59 \%$, case (I)) and Ptasinski et al. (2003) (symbol: $\diamond \mathrm{DR}=40 \%$, case $(\mathrm{N})$; $\bigcirc \mathrm{DR}=61 \%$, case $(\mathrm{L})$, and $\times \mathrm{DR}=64 \%$, case $(\mathrm{O})$ ) and predictions (continuum lines against to Ptasinski et al. (2003) data, and dash lines against to Iaccarino et al. (2010) data), for channel turbulent flow, with rheological parameters defined in Table 2 and 3. Each colour corresponds to a specific DR.

the equilibrium state is achieved through the 3 parameter, where the viscoelastic part become null for Newtonian fluids.

$\nu_{T}=C_{\mu} \times f_{\mu} \times \frac{k^{2}}{\tilde{\varepsilon}^{N}}$

with

$f_{\mu}=\left[1-\exp \left(-\frac{y^{+}}{26.5\left(1+0.0165 \times\left(\frac{C_{k k}-3}{L^{0.75}(\beta / 0.9)^{6 \beta}}\right)^{1.27}\right)}\right)\right]^{2}$ and $C_{\mu}=0.09$

where $y^{+}=u_{\tau} y / \nu_{\text {wall }}$, based on the wall viscosity of the solution $v_{\text {wall }}=v_{s}+v_{\tau_{p, \text { wall }}}$, sum of solvent viscosity with the polymeric viscosity at the wall, $\nu_{\tau_{p, \text { wall }}}=\bar{\tau}_{x y \text {,wall }}^{p} /(\rho \dot{\gamma})$, where $\dot{\gamma}$ is the shear rate.

The remaining viscoelastic terms, the viscoelastic stress work $\left(\varepsilon^{V}\right)$ and the viscoelastic destruction term, $E_{\tau_{p}}$, of the transport equation of $k$ and $\tilde{\varepsilon}^{N}$, are modelled using the same closures developed by Pinho et al. (2008) and Resende et al. (2011), respectively. The viscoelastic stress work is given by

$\varepsilon^{V} \equiv \frac{1}{\rho} \overline{\tau_{i k, p}^{\prime} \frac{\partial u_{i}}{\partial x_{k}}}=\frac{\eta_{p}}{\rho \lambda}\left[C_{i k} \overline{f\left(C_{m m}+c_{m m}\right) \frac{\partial u_{i}}{\partial x_{k}}}+\overline{c_{i k} f\left(C_{m m}+c_{m m}\right) \frac{\partial u_{i}}{\partial x_{k}}}\right]$.

Initially, based on the DNS data, Pinho et al. (2008) verified that the double correlation can be neglected by comparison with the triple correlation at low DR regime, and later it was also confirmed for intermediate and high DR regimes, (Resende et al., 2011; Masoudian et al., 2013). Pinho et al. (2008) also showed that in low drag reduction regime the triple correlation can be decoupled and modelled as function of $N L T_{m m} / 2$, Eq. (32). Recently, Masoudian et al. (2015) demonstrated that the model is capable to predict all the individual components with good performance within $5 \%$ error. 
Table 2

Description of the DNS parameters for turbulent channel flow at $\operatorname{Re}_{\tau 0}=300$, Iaccarino et al. (2010).

\begin{tabular}{|c|c|c|c|c|c|c|c|}
\hline \multirow[t]{2}{*}{ Case } & \multicolumn{3}{|c|}{ Rheological parameters } & \multicolumn{3}{|c|}{ Drag reduction (\%) } & \multirow[t]{2}{*}{ Error (\%) } \\
\hline & $W i_{\tau 0}$ & $\beta$ & $L^{2}$ & DNS & Iaccarino et al. (2010) & Present model & \\
\hline (F) & 36 & 0.9 & 3600 & 33 & 31 & 34 & 3.0 \\
\hline (G) & 60 & 0.9 & 3600 & 47 & 47 & 46 & 2.1 \\
\hline$(\mathrm{H})$ & 36 & 0.9 & 10,000 & 35 & 34 & 32 & 8.6 \\
\hline (I) & 120 & 0.9 & 10,000 & 59 & 62 & 58.2 & 1.4 \\
\hline$(\mathrm{J})$ & 36 & 0.9 & 19,600 & 32 & 34 & 27.6 & 13.8 \\
\hline (K) & 60 & 0.9 & 19,600 & 42 & 46 & 48 & 14.3 \\
\hline
\end{tabular}

Table 3

Description of the DNS parameters for turbulent channel flow at $\operatorname{Re}_{\tau 0}=180$, Ptasinski et al. (2003).

\begin{tabular}{|c|c|c|c|c|c|c|c|}
\hline \multirow[t]{2}{*}{ Case } & \multirow[t]{2}{*}{ Ptasinski et al. (2003) } & \multicolumn{3}{|c|}{ Rheological parameters } & \multicolumn{2}{|c|}{ Drag reduction (\%) } & \multirow[t]{2}{*}{ Error (\%) } \\
\hline & & $W i_{\tau 0}$ & $\beta$ & $L^{2}$ & DNS & Present model & \\
\hline (L) & Run B & 54 & 0.6 & 1000 & 61 & 58 & 4.9 \\
\hline (M) & Run C & 72 & 0.6 & 1000 & 66 & 61.7 & 6.5 \\
\hline$(\mathrm{N})$ & Run D & 54 & 0.8 & 1000 & 40 & 43.6 & 9.0 \\
\hline (O) & Run E & 54 & 0.4 & 1000 & 64 & 64 & 0 \\
\hline
\end{tabular}

Table 4

Description of the DNS parameters for turbulent channel flow at $\operatorname{Re}_{\tau 0}=125$, Li et al. (2006).

\begin{tabular}{|c|c|c|c|c|c|c|}
\hline \multirow[t]{2}{*}{ Case } & \multicolumn{3}{|c|}{ Rheological parameters } & \multicolumn{2}{|c|}{ Drag reduction (\%) } & \multirow[t]{2}{*}{ Error (\%) } \\
\hline & $W i_{\tau 0}$ & $\beta$ & $L^{2}$ & Li et al. (2006) & Present model & \\
\hline (P) & 50 & 0.9 & 900 & 31 & 34.5 & 11.3 \\
\hline (Q) & 100 & 0.9 & 900 & 37 & 39 & 5.4 \\
\hline (R) & 100 & 0.9 & 3600 & 56.5 & 51 & 9.7 \\
\hline (S) & 100 & 0.9 & 7200 & 69 & 54.9 & 20 \\
\hline
\end{tabular}

Table 5

Description of the DNS parameters for turbulent channel flow at $\operatorname{Re}_{\tau 0}=180$, Li et al. (2006).

\begin{tabular}{|c|c|c|c|c|c|c|}
\hline \multirow[t]{2}{*}{ Case } & \multicolumn{3}{|c|}{ Rheological parameters } & \multicolumn{2}{|c|}{ Drag reduction (\%) } & \multirow[t]{2}{*}{ Error $(\%$} \\
\hline & $W i_{\tau 0}$ & $\beta$ & $L^{2}$ & Li et al. (2006) & Present model & \\
\hline$(\mathrm{T})$ & 50 & 0.9 & 900 & 30.5 & 33.9 & 11.1 \\
\hline (U) & 100 & 0.9 & 900 & 38.5 & 38.7 & 0.5 \\
\hline (V) & 100 & 0.9 & 3600 & 54 & 51 & 5.5 \\
\hline (X) & 100 & 0.9 & 14,400 & 71 & 58 & 18 \\
\hline
\end{tabular}

$$
\begin{aligned}
\varepsilon^{V} & \approx \frac{\eta_{p}}{\rho \lambda}\left[\overline{c_{i k} f\left(C_{m m}+c_{m m}\right) \frac{\partial u_{i}}{\partial x_{k}}}\right] \approx \frac{\eta_{p}}{\rho \lambda}\left[f\left(C_{m m}\right) \overline{c_{i k} \frac{\partial u_{i}}{\partial x_{k}}}\right] \\
& \approx \frac{\eta_{p}}{\rho \lambda} f\left(C_{m m}\right) \frac{N L T_{m m}}{2}
\end{aligned}
$$

The model used here for $E_{\tau_{p}}$ is given by Eq. (33) and assumed that it depends on the same quantities as the classical Newtonian destruction term of the transport equation of $\varepsilon$, i.e., proportional to $\varepsilon^{2} / k$. This model is based on the Resende et al. (2011) closure but more simple and capable to capture the solvent ratio variations, close and away from the wall.

$$
\begin{aligned}
E_{\tau_{p}} & =2 \eta_{s} \frac{\eta_{p}}{\lambda\left(L^{2}-3\right)} \overline{\frac{\partial u_{i}}{\partial x_{m}}} \frac{\partial}{\partial x_{k}}\left\{\frac{\partial}{\partial x_{m}}\left[f\left(C_{n n}\right) f\left(\widehat{C}_{p p}\right) c_{q q} C_{i k}\right]\right\} \\
& \approx-2 \beta(1-\beta) \frac{\tilde{\varepsilon}^{N 2}}{k}\left[C_{\varepsilon F 1} \times \frac{\varepsilon^{V}}{\tilde{\varepsilon}^{N}}+C_{\varepsilon F 2} \times L^{1.1} \times f\left(C_{m m}\right)\right]
\end{aligned}
$$

where the coefficients parameters are $C_{\varepsilon F 1}=1.25$ and $C_{\varepsilon F 2}=0.045 \times\left(25 / W i_{\tau 0}\right)^{0.6}$.

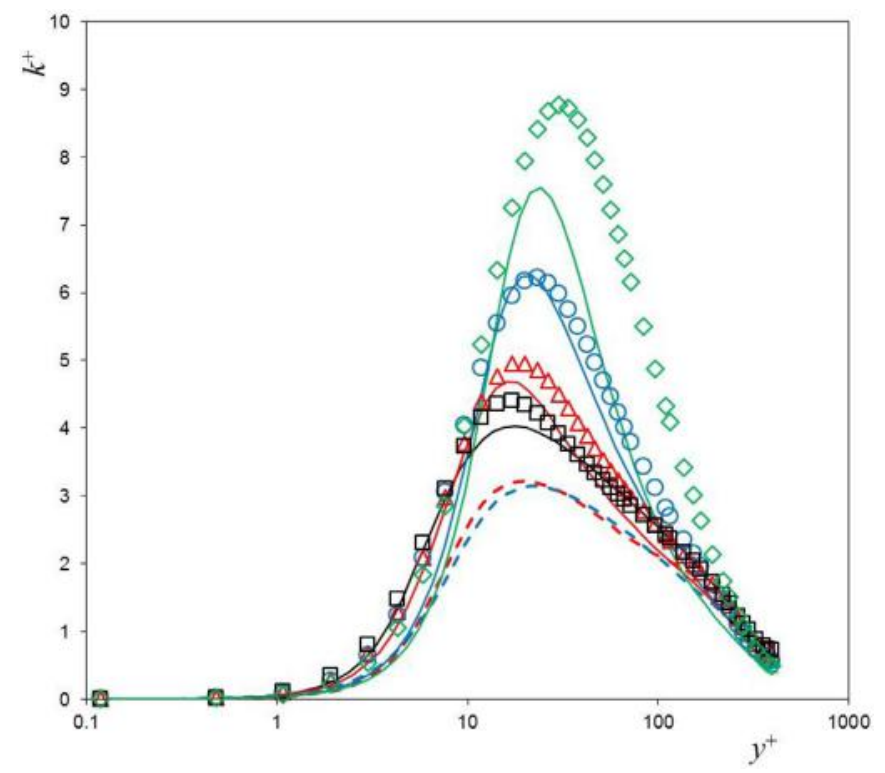

Fig. 4. Comparison of turbulent kinetic energy between DNS data (symbols: $\square$ Newtonian; $\Delta \mathrm{DR}=18 \%$, case (A); $O \mathrm{DR}=37 \%$, case (B), and $\diamond \mathrm{DR}=51 \%$, case (C)) and predictions with the new model (continuum lines), and previous model (dash lines). Each colour corresponds to a specific DR.

\section{Summary of the present model}

The governing and the transport model equations are given below, using the closures developed in the previous subsection.

Momentum equation:

$\rho \frac{D U_{i}}{D t}=-\frac{\partial \bar{p}}{\partial x_{i}}+\frac{\partial}{\partial x_{k}}\left[\left(\eta_{s}+\nu_{T}\right) \frac{\partial U_{i}}{\partial x_{k}}\right]+\frac{\partial}{\partial x_{k}}\left(\frac{\eta_{p}}{\lambda}\left[f\left(C_{k k}\right) C_{i j}-\delta_{i j}\right]\right)$

where the eddy viscosity is given by Eq. (29) and Eq. (30).

Conformation tensor equation:

$\frac{D C_{i j}}{D t}-M_{i j}-N L T_{i j}=-\frac{1}{\lambda}\left[f\left(C_{k k}\right) C_{i j}-\delta_{i j}\right]$

with 


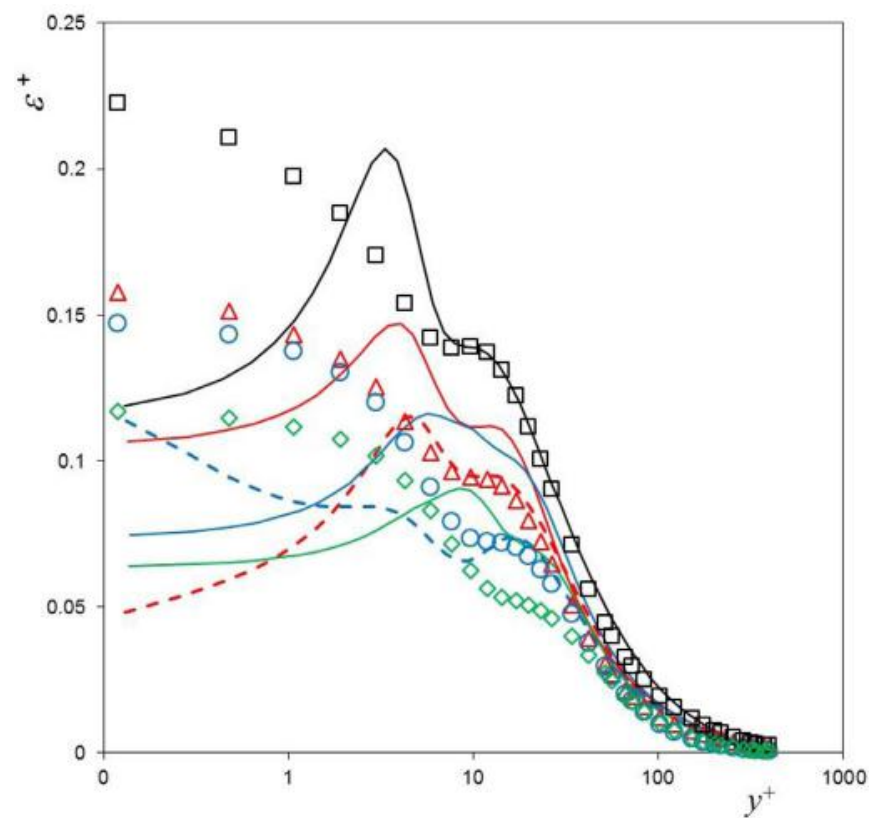

Fig. 5. Comparison of the rate of Newtonian dissipation of $k$ between DNS data (symbols: $\square$ Newtonian; $\Delta \mathrm{DR}=18 \%$, case (A); $\bigcirc \mathrm{DR}=37 \%$, case (B), and $\diamond$ $\mathrm{DR}=51 \%$, case $(C)$ ) and predictions with the new model (continuum lines), and previous model (dash lines). Each colour corresponds to a specific DR.

$$
\begin{aligned}
N L T_{i j} \approx & f_{N 1} C_{N 1} \frac{\lambda \times L^{0.42} \times \varepsilon^{N}}{v_{0} \times f\left(C_{m m}\right)^{0.8}} \delta_{i j}-f_{N 1}^{0.2} \frac{C_{N 2}}{L^{0.15}}\left[C_{j k} \frac{\partial U_{i}}{\partial x_{k}}+C_{i k} \frac{\partial U_{j}}{\partial x_{k}}\right]+ \\
& +f_{N 1}^{0.9} f_{N 2} C_{N 3}\left(\frac{C_{m m} f_{\mathrm{Re}}}{(\beta / 0.9)^{0.7 \times \beta}}\right)^{0.7} \frac{\frac{\partial U_{i}}{\partial x_{k}} \frac{\partial U_{j}}{\partial x_{k}}}{\sqrt{\frac{\partial U_{p} \partial U_{p}}{\partial x_{q}}}}
\end{aligned}
$$

where the damping function are defined by Eq. (21) to Eq. (23), and the viscoelastic parameters are presented after.

Transport equation of $k$ :

$\rho \frac{\partial k}{\partial t}+\rho U_{i} \frac{\partial k}{\partial x_{i}}=\frac{\partial}{\partial x_{i}}\left[\left(\eta_{s}+\frac{\rho f_{t} \nu_{T}}{\sigma_{k}}\right) \frac{\partial k}{\partial x_{i}}\right]-\rho \overline{u_{i} u_{k}} \frac{\partial U_{i}}{\partial x_{k}}-\rho\left(\tilde{\varepsilon}^{N}+D\right)-\rho \varepsilon^{V}$

with

$D=2 v_{s}\left(\frac{d \sqrt{k}}{d x_{i}}\right)^{2}$ and $\varepsilon^{V} \approx \frac{\eta_{p}}{\rho \lambda} f\left(C_{m m}\right) \frac{N L T_{m m}}{2}$

Dissipation transport equation:

$$
\begin{aligned}
\frac{\partial \rho \tilde{\varepsilon}^{N}}{\partial t}+\frac{\partial \rho U_{i} \tilde{\varepsilon}^{N}}{\partial x_{i}}= & \frac{\partial}{\partial x_{i}}\left[\left(\eta_{s}+\frac{\rho f_{t} \nu_{T}}{\sigma_{\varepsilon}}\right) \frac{\partial \tilde{\varepsilon}^{N}}{\partial x_{i}}\right]+f_{1} C_{\varepsilon_{1}} \frac{\tilde{\varepsilon}^{N}}{k} P_{k}-f_{2} C_{\varepsilon_{2}} \rho \frac{\tilde{\varepsilon}^{N^{2}}}{k} \\
& +\rho E+E_{\tau_{p}}
\end{aligned}
$$

with

$$
\begin{aligned}
E & =\nu_{s} \nu_{T}\left(1-f_{\mu}\right)\left(\frac{\partial^{2} U_{j}}{\partial x_{i} \partial x_{k}}\right)^{2} \text { and } E_{\tau_{p}} \\
& \approx-2 \beta(1-\beta) \frac{\tilde{\varepsilon}^{N 2}}{k}\left[C_{\varepsilon F 1} \times \frac{\varepsilon^{V}}{\tilde{\varepsilon}^{N}}+C_{\varepsilon F 2} \times L^{1.1} \times f\left(C_{m m}\right)\right]
\end{aligned}
$$

The remaining damping functions and coefficients associated to the Newtonian part are described below Eq. (18).

\section{Results and discussion}

In this section, the performance of the present model is evaluated at fully-developed channel flows for different Reynolds and Weisenberg numbers, for a wide variety of rheological parameters, and comparing against the DNS data. The numerical simulations were carried out using a finite-volume code for the FENE-P fluids (Resende et al., 2011), with non-uniform meshes containing 99 cells to cover the channel width. About 10 cells were located inside each of the viscous sublayers in order to provide mesh independent results, with errors within $0.5 \%$ for the mean velocity and the friction factor. No-slip boundary conditions were applied at the wall with $U=0, k=0$ and $\tilde{\varepsilon}^{N}=0$.

Fig. 2 and Fig. 3 compare the predicted velocity profiles with DNS data for a large set of data covering all regimes of DR with different values of $\beta, L^{2}$, Reynolds numbers, and Weissenberg numbers, including also the predictions used for calibration and from the previous model of Resende et al. (2011). The DNS rheological parameters and flow conditions are defined in Tables 1-3, for different Reynolds numbers, $\operatorname{Re}_{\tau 0}=395, \operatorname{Re}_{\tau 0}=300$ and $\operatorname{Re}_{\tau 0}=180$, respectively, together with the DR predictions. All the profiles across the channel are in good agreement with DNS data. The mean velocity in the viscous sublayer collapse to the $u^{+}=y^{+}$definition, and further away from the wall, in the logarithmic layer, the profile is shifted upwards parallel to the logarithmic profile of the Newtonian flows, according to a drag reduction characteristic at low DR regime, found in the experimental and DNS results. Furthermore, the velocity of the polymeric flow increases, and consequently the upward shift of the logarithmic profile occur, which is usual interpreted as a thickening of the buffer layer. At maximum drag reduction the model is not capable to keep the same performance, as observed in case (E) of Table 1, which can be justified by the isotropic assumption of the turbulence model. To extend the present model to Reynolds number and solvent ratio variations, it was used the DNS data of Iaccarino et al. (2010), case (F) of Table 2, and Ptasinski et al. (2003), case (O) of Table 3, respectively, for calibration, and the remaining cases presented are to assess the model performance. The Iaccarino et al. (2010) predictions are presented in Table 2 for comparison. Note that the DNS simulations of Ptasinski et al. (2003) were performed at $\mathrm{Re}_{\tau 0}=360$ which differ from the present work, because their Reynolds definition is based on total height of the channel, but here is considered half-height of the channel equivalent to $\operatorname{Re}_{\tau 0}=180$. A turbulent viscoelastic model should be capable to predict the viscoelastic behavior with different combinations of four parameters $\left(W i_{\tau_{0}}, \beta, L^{2}\right.$ and $\left.R e_{\tau_{0}}\right)$, which clearly demonstrates the complexity of the subject. In this case, the model was calibrated for the most critical situation, $\beta=0.4$, and tested for the remaining cases. However, it was found that for $\beta=0.8$ the error increased, but decreasing for $\beta=0.9$ (see Table 1), which means that the error does not change linearly. Also, Tsukahara and Kawaguchi (2013), using the Giesekus model, developed of a $k-\varepsilon$ turbulence model for viscoelastic fluids, however it failed to predict correctly the drag reduction for lower values of Weissenberg numbers and $\beta$. In the worst case tested, the model predicted $1 \%$ of DR, whereas the DNS data shows $23 \%$. For low Reynolds numbers, $\operatorname{Re}_{\tau 0}=125$ and $\operatorname{Re}_{\tau 0}=180$, the predictions start to apart from the DNS data, Table 4 and Table 5, specially at low regime of DR, which can be justified by the low accuracy of the Newtonian turbulence model used for the solvent part. The prediction error of the Darcy friction factor for a Newtonian case at $\operatorname{Re}_{\tau 0}=125$ is about $6.6 \%$. At maximum drag reduction, the prediction error increases, showing the limitations of this type of model, due to the use of isotropic assumptions, where the flow at this stage is clearly anisotropic.

Besides of the present model capacity to predict all regimes of DR with good performance, another main advantage can be observed in Fig. 4, which shows the turbulent kinetic energy behaviour. The predictions are capable to capture correctly the $k$ evolution, the increase of the maximum value and its shift away from the wall with the increase of drag reduction, when comparing with the DNS data. This improvement is associated with the inclusion of a polymeric contribution in the eddy viscosity model, through the damping function, $f_{\mu}$ Eq. (30). As mention before this damping function acts close to the wall, especially 

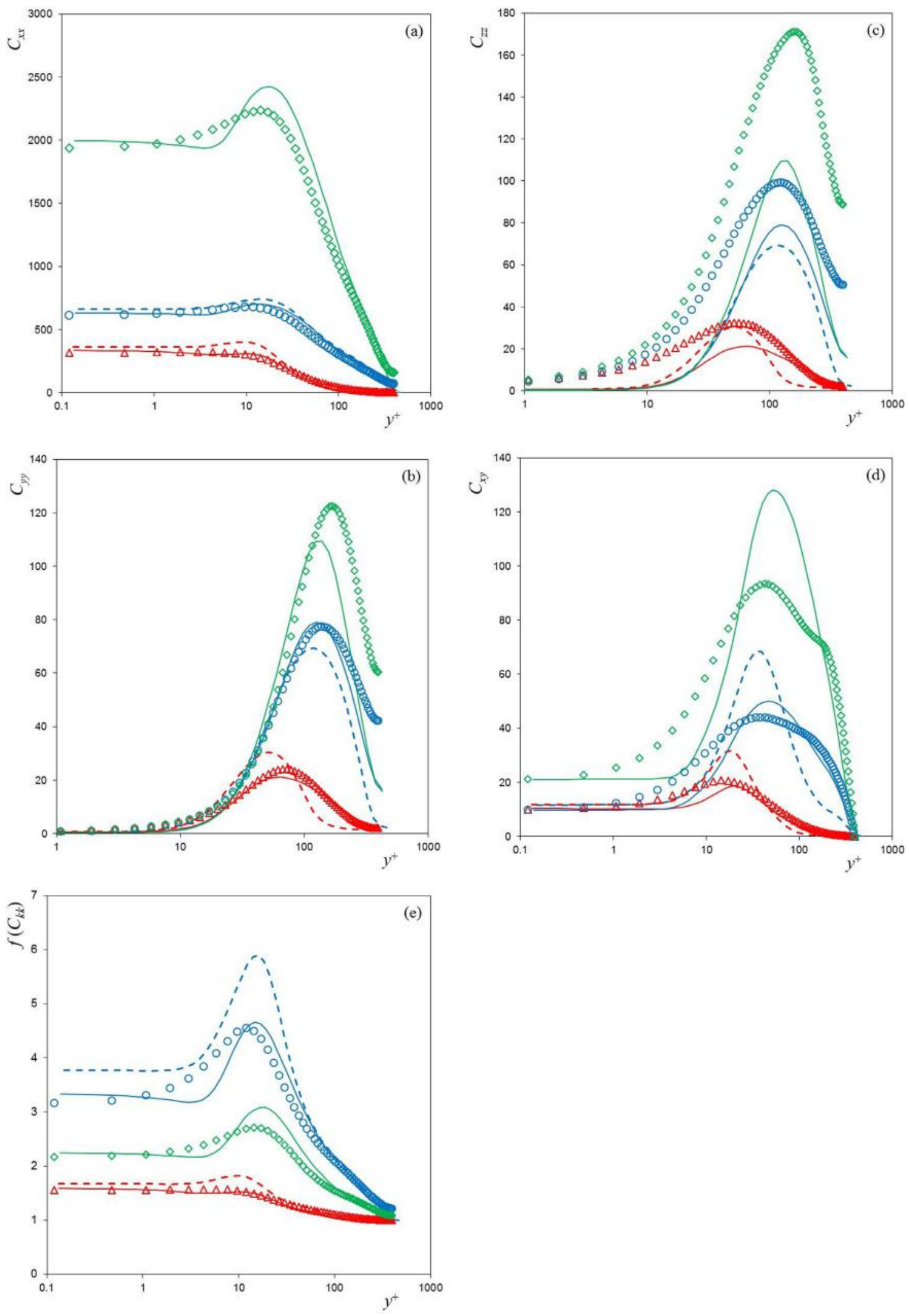

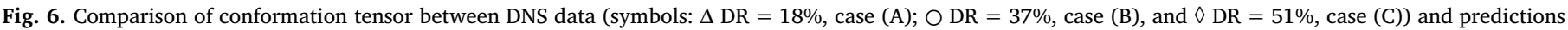

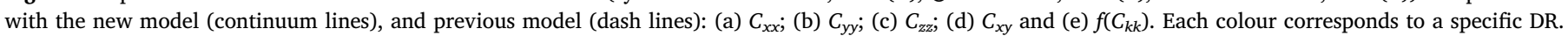

into the viscous sublayer, but its impact in the energy cascade is high, allowing the energy to propagate across the channel and increase the turbulent kinetic energy, important to achieve higher values of DR. In addition, it is possible to observe that the predictions from previous model presented an opposite trend, decrease of the maximum value of $k$ $\left(k^{+}=3.2\right.$ for $\mathrm{DR}=18 \%$ and $k^{+}=3.1$ for $\left.\mathrm{DR}=37 \%\right)$ below the peak 
value for the Newtonian case, $k^{+}=4$. This incorrect behaviour demonstrates some limitations in the previous model, therefore, it can not achieve the maximum drag reduction due to the excessive reduction of $k$. For high regime of DR the predictions started to apart from the DNS data, underpredicting the peak value of $k$, which means that the reduction of the eddy viscosity through $f_{\mu}$ is insufficient to reduce the shear Reynolds stress, so it is completed by a decrease of $k$. Even so, the influence of $k$ reduction on DR prediction, for $\mathrm{DR}=51 \%$, is low, about $14 \%$, by comparison of the maximum value of $k$ between the predictions and DNS, but as the DR increases, this effect becomes more intense. An alternative is the use of a higher level turbulence model, as made by Masoudian et al. (2015), but this is beyond the scope of this work.

The predictions of the normalised rate of dissipation of $k$ are plotted in Fig. 5 and show an overprediction of the plateau close to the wall. However, a proportional reduction of the plateau values with the increase of DR is achieved, with similar behaviour at the wall, where it can also be observed the saturation effect for higher DR. The corresponding predictions of the previous model capture correctly the behaviour close to the wall, but presented some instabilities at the wall, which also occur here for the case of $37 \%$ of DR, where the plateau is not well defined. Away from the wall, the predictions of $\tilde{\varepsilon}^{N}$ are good due to the introduction of the viscoelastic destruction term, $E_{\tau_{p}}$, in the transport equation of $\tilde{\varepsilon}^{N}$, which decreases $\tilde{\varepsilon}^{N}$ as flow viscoelasticity increases. The main advantage of the closure presented here is the complexity reduction of Resende et al. (2011) model, keeping the same predictions accuracy, specially in the logarithmic region. The inclusion of a viscoelastic destruction closure also was made by Masoudian et al. (2013) to improve the $k-\varepsilon-\overline{v^{2}}-f$ turbulence model of Iaccarino et al. (2010), for FENE-P fluids, which allowed to increase the performance of the model in terms of the Newtonian dissipation predictions at the logarithmic region. The maximum value of $\tilde{\varepsilon}^{N}$ and its location are incorrectly predicted, consequence of the Newtonian closures, which is a known defect of the $k-\varepsilon$ model.

The evolution of the conformation tensor is well predicted for all components, Fig. 6(a)-(e), with exception for the $z z$ component due to the isotropic assumption used to modelling the $N L T_{i j}$ term, introducing a direct impact in the conformation tensor predictions. The quality of the $C_{i j}$ predictions is a consequence of the $N L T_{i j}$ closure performance since $N L T_{i j}$ is the major contribution to the balance of $C_{i j}$. As can be seen in Fig. 6(a) and (b), the predictions of $C_{y y}$ and $C_{x x}$ show a good agreement with the DNS data, with the exception of $C_{z z}$, where there is an underprediction, however its impact is not significant. The major contribution for the trace of the conformation tensor is related to the $C_{x x}$, and it can be visualized through the $f\left(C_{k k}\right)$ function in Fig. 6(e). For high regime of DR there is a small overprediction of the maximum value of $f\left(C_{k k}\right)$ due to the $C_{x x}$ overprediction, even so the region of high chain dumbbell extension is limited to the near wall region $\left(y^{+}<50\right)$, which is in concordance with findings of Li et al. (2006). Regarding $C_{y y}$ predictions, a good performance is required because it acts directly to the shear component of the conformation tensor, $C_{x y}$, and as can be observed in Fig. 6(d), the predictions are capable to capture the behaviour for low and intermediate regime of DR, with exception at high regime where there is an overprediction of the maximum value, however, its location is correctly predicted. Overall, it is evident that the conformation tensor predictions are capable to capture all the main features with good performance and better quality predictions, when compared with the previous model of Resende et al. (2011), consequence of the new $N L T_{i j}$ model developed in the present work.

\section{Conclusions}

The low-Reynolds-number $k-\varepsilon$ turbulence model of Resende et al. (2011) for dilute polymeric solutions, represented by the FENE-P rheological constitutive equation, is improved up to maximum drag reduction. The turbulence model is tested against DNS data, to account the DR variations, with the different combinations of the viscoelastic rheological parameters of the relaxation time of the fluid, the maximum molecular extensibility of the model dumbbell and the ratio of the solvent, where four sets of DNS were used for calibration. The present model is capable to predict all regimes of DR, in fully-developed channel turbulent flow, with good performance.

The main feature is the inclusion of the viscoelastic effect through the damping function of the eddy viscosity, to consider the elastic proprieties close to the wall and correct the energy cascade distribution, where the small scales redistributed the energy to the large scales. This is accomplished by reducing the eddy viscosity, proportional to the DR increase. The correct behaviour close to the wall allows the proper prediction of the turbulent kinetic energy, the increase of the maximum value with the DR increase, in contrast with the predictions of the previous model of Resende et al. (2011), where was unable to reach the high regime of DR.

Other improvements were implmented to the Reynolds-averaged nonlinear term of the polymer conformation equation (denoted $N L T_{i j}$ ) and to the viscoelastic destruction term of the rate of dissipation of $k$ by the Newtonian solvent, previous developed by Resende et al. (2011). The improved $N L T_{i j}$ model present better performance for all regimes of $\mathrm{DR}$, when compared with the previous model, but the main advantage is the reduction of number of terms and the capacity to predict the negative part, which acts like production term in the transport equation of $k$. This develoment also eliminates the necessity of damping functions depending on the wall distance, and the dependence of the friction velocity through the Weissenberg number, given more stability in $3 \mathrm{D}$ simulations. In terms of the viscoelastic destruction term, Tsukahara and Kawaguchi (2013) reported that the most serious deficiency found in their model was the overpredicted dissipation rate, given an underestimated viscoelastic contribution, consequent of neglecting this term. For this reason, a simpler model of Resende et al. (2011) for the viscoelastic destruction term is proposed here, keeping the same predictions accuracy of $\varepsilon$ in the logarithmic region, while close to the wall there is an overprediction of the plateau, however, it is capable to capture the saturation effect with the increase of DR.

Overall, the predictions compare very well with DNS data in terms of mean velocity, turbulent kinetic energy and dissipation, and is capable to capture all viscoelastic features at all ranges of drag reduction, for different Reynolds numbers. The main advantage of the present model is the combination of the performance with simplicity, which will allow the easy implementation in 3D codes.

\section{Acknowledgements}

Financial support provided by FAPESP - Fundação de Amparo à Pesquisa do Estado de São Paulo through Project N ${ }^{\circ} 2013 / 01521-4$ and CNPq - Conselho Nacional de Desenvolvimento Científico e Tecnológico through Project $N^{\circ} 449361 / 2014-4$, is gratefully acknowledged by P. R. Resende.

\section{References}

Angelis, E.D., Casciola, C.M., Piva, R., 2002. DNS of wall turbulence: dilute polymers and self-sustaining mechanisms. Comp. Fluids 31, 495-507.

Angelis, E.D., Casciola, C.M., L'vov, V.S., Pomyalov, A., Procaccia, I., Tiberkevich, V. 2004. Drag reduction by a linear viscosity profile. Phys. Rev. E. 70, 055301-055301055301-055304.

Bird, R.B., Dotson, P.J., Johnson, N.L., 1980. Polymeric solution rheology based on a finitely extensible bead-spring chain model. J. Non-Newt. Fluid Mech 7, 433-468.

Cruz, D.O.A., Pinho, F.T., Resende, P.R., 2004. Modeling the new stress for improved drag reduction predictions of viscoelastic pipe flow. J. Non Newtonian Fluid Mech. 121, $127-141$.

Dallas, V., Vassilicos, J.C., Hewitt, G.F., 2010. Strong polymer-turbulence interactions in viscoelastic turbulent channel flow. Phys. Rev. E. 82, 066303-066301-066303066319.

Housiadas, K.D., Beris, A.N., Handler, R.A., 2005. Viscoelastic effects on higher order 
statistics and coherent structures in turbulent channel flow. Phys. Fluids 17. Iaccarino, G., Shaqfeh, E.S., Dubief, Y., 2010a. Reynolds-averaged modeling of polymer drag reduction in turbulent flows. J. Non Newtonian Fluid Mech. 165, 376-384.

Iaccarino, G., Shaqfeh, E.S.G., Dubief, Y., 2010b. Reynolds-averaged modeling of polymer drag reduction in turbulent flows. J. Non Newtonian Fluid Mech. 165, 376-384.

Kim, K., Li, C.F., Sureshkumar, R., Balachandar, S., Adrian, R., 2007. Effects of polymer stresses on eddy structures in drag-reduced turbulent channel flow. J. Fluid Mech. 584, 281-299.

L'vov, V.S., Pomyalov, A., Procaccia, I., Tiberkevich, V., 2004. Drag reduction by polymers in wall bounded turbulence. Phys. Rev. E. 92, 244503-244501-244503-244504.

Leighton, R.I., Walker, D.T., Stephens, T.R., Garwood, G.C., 2003. Reynolds stress modeling for drag-reducing viscoelastic flow. In: Joint ASME/ JSME Fluids Engineering Symposium on Friction Drag Reduction. Honolulu, Hawai, USA.

Li, C.F., Gupta, V.K., Sureshkmar, R., Khomami, B., 2006a. Turbulent channel flow of dilute polymeric solutions: drag reduction scaling and an eddy viscosity model. J. Non Newtonian Fluid Mech. 139, 177-189.

Li, C.F., Sureshkmar, R., Khomami, B., 2006b. Influence of rheological parameters on polymer induced turbulent drag reduction. J. Non Newtonian Fluid Mech. 140, $23-40$.

Lumley, J.L., 1969. Drag reduction by additives. Annu. Rev. Fluid Mech 1, 367-384.

Lumley, J.L., 1973. Drag reduction in turbulent flow by polymer additives. J. Poly. Sci. 7, 263-290.

Masoudian, M., Kim, K., Pinho, F.T., Sureshkumar, R., 2013. A viscoelastic $k-e-v^{2}-f$ turbulent flow model valid up to the maximum drag reduction limit. J. Non Newtonian Fluid Mech. 202, 99-111.

Masoudian, M., Kim, K., Pinho, F.T., Sureshkumar, R., 2015. A Reynolds stress model for turbulent flow homogeneous polymer solutions. Int. J. Heat Fluid Flow 54, 220-235.

Min, T., Yoo, J.Y., Choi, H., Joseph, D.D., 2003. Drag reduction by polymer additives in a turbulent channel flow. J. Fluid Mech. 486, 213-238.

Nagano, Y., Hishida, M., 1987. Improved form of the $k-e$ model for wall turbulent shear flows. J. Fluids Eng. 109, 156-160.

Nagano, Y., Shimada, M., 1993. Modeling the dissipation-rate equation for two-equation turbulence model. In: Ninth symposium on "Turbulent shear flows". Kyoto, Japan.

Park, T.S., Sung, H.J., 1995. A nonlinear low-Reynolds-number $k$-e model for turbulent separated and reattaching flows-I. Flow field comptations. Int. J. Heat Mass Transf. $38,2657-2666$.

Pinho, F.T., Li, C.F., Younis, B.A., Sureshkumar, R., 2008. A low Reynolds number k-e turbulence model for FENE-P viscoelastic fluids. J. Non Newtonian Fluid Mech. 154, 89-108.

Pinho, F.T., 2003. A GNF framework for turbulent flow models of drag reducing fluids and proposal for a k-e type closure. J. Non Newtonian Fluid Mech. 114, 149-184.

Ptasinski, P.K., Boersma, B.J., Nieuwstadt, F.T.M., Hulsen, M.A., Brule, B.H.A.A.V.D., Hunt, J.C.R., 2003. Turbulent channel flow near maximum drag reduction: simulation, experiments and mechanisms. J. Fluid Mech. 490, 251-291.

Resende, P.R., Escudier, M.P., Presti, F., Pinho, F.T., Cruz, D.O.A., 2006. Numerical predictions and measurements of Reynolds normal stresses in turbulent pipe flow of polymers. Int. J. Heat Fluid Flow 27, 204-219.

Resende, P.R., Kim, K., Younis, B.A., Sureshkumar, R., Pinho, F.T., 2011. A FENE-P $k-\varepsilon$; turbulence model for low and intermediate regimes of polymer-induced drag reduction. J. Non Newtonian Fluid Mech. 166, 639-660.

Resende, P.R., Pinho, F.T., Cruz, D.O.A., 2013a. A Reynolds stress model for turbulent flows of viscoelastic fluids. J. Turbul. 14, 1-36.

Resende, P.R., Pinho, F.T., Kim, K., Younis, B.A., Sureshkumar, R., 2013b. Development of a low-Reynolds-number $k-\omega$ model for FENE-P Fluids. Flow Turbul. Combust. 90 69-94.

Resende, P.R., Cavadas, A.S., 2018. New developments in isotropic turbulent models for FENE-P fluids. Fluid Dynamics Research 50, 025508.

Tabor, M., Gennes, P.G.D., 1986. A cascade theory of drag reduction. Europhys. Lett. 2, 519-522.

Thais, L., Gatski, T.B., Mompean, G., 2013. Analysis of polymer drag reduction mechanisms from energy budgets. Int. J. Heat Fluid Flow 43, 52-61.

Tsukahara, T., Kawaguchi, Y., 2013. Proposal of damping function for low-Reynoldsnumber k-e model applicable in prediction of turbulent viscoelastic-fluid flow. J. Appl. Math. 2013, 1-15. 